\title{
Ribosomal DNA Restriction Analysis Reveals Genetic Heterogeneity in Saccharomyces cerevisiae Meyen ex Hansen
}

\author{
FRANCIS I. MOLINA, ${ }^{1 *}$ TORU INOUE, ${ }^{2}$ AND SHUNG-CHANG JONG ${ }^{1}$ \\ American Type Culture Collection, Rockville, Maryland 20852, ${ }^{1}$ and Yamazaki Baking Co., \\ Ltd., Tokyo, Japan ${ }^{2}$
}

\begin{abstract}
A ribosomal DNA restriction analysis of 17 strains belonging to the Saccharomyces cerevisiae complex revealed two major groups, one of which corresponded to Saccharomyces bayanus. Our results generally correlate with previously reported genetic and molecular data and support the conclusion that $S$. bayanus should be reinstated as a separate taxon.
\end{abstract}

The schemes currently used for classifying yeasts in the genus Saccharomyces are based largely on physiological features, such as fermentation reactions and resistance to cycloheximide $(3,29)$. The use of these characteristics is necessitated by the paucity of morphological criteria. However, mounting evidence suggests that such characteristics may not always be adequate for species delimitation, and interstrain variability has been observed $(3,7,18,21,29)$. The instability of distinguishing characteristics $(22,23)$ and the apparent absence of reproductive isolation (13) have led to the relegation of many previously recognized Saccharomyces species to physiological races of Saccharomyces cerevisiae Meyen ex Hansen $(24,29,30)$. To resolve the taxonomic ambiguities that result from variable biochemical reactions, other criteria have been used for species delimitation within this genus. These criteria include serological properties (5), levels of DNA relatedness $(4,10,21,26,30)$, restriction endonuclease patterns (17), and electrophoretic karyotyping (8). According to Vaughan-Martini and Martini (27), most Saccharomyces species can be differentiated only by using DNA-DNA hybridization data. On the basis of DNA reassociation results, these authors recognized $S$. bayanus and $S$. pastorianus as species that are distinct from $S$. cerevisiae, a view shared by Banno and Kaneko (2).

Because of the presence of both highly conserved and variable regions, rRNA sequences have been used as a tool for analyzing phylogenetic relationships $(10,11)$. In this study, we examined restriction fragment length polymorphisms in the gene that codes for the small-subunit ribosomal DNA (rDNA), and we obtained further evidence of genetic heterogeneity in $S$. cerevisiae. We also found that restriction patterns could be used to assign most of the strains which we examined to either $S$. cerevisiae or $S$. bayanus. The original specific epithets are used below.

We studied 17 strains (Table 1) of organisms that were considered to be either synonyms or physiological races of $S$. cerevisiae $(3,29,30)$. $S$. kluyveri, a species that is thought to be an outlying member of the genus (10), was included for comparison. The procedure used for DNA isolation and the conditions used for the polymerase chain reaction (PCR) have been described previously (12). The primers which we used were those described by White et al. (28) and had the following sequences: primer NS1, 5'-GTAGTCATATGCTT GTCTC; and primer NS8, 5'-TCCGCAGGTTCACCTAC GGA. The PCR products were digested with the following enzymes: AciI, Cfr13I, DdeI, HhaI, HinfI, MspI, PalI, RsaI,

\footnotetext{
* Corresponding author.
}

Sau3AI, ScrfI, and Taq $\alpha$ I. The restriction fragments were electrophoresed on 2.6\% NuSieve 3:1 agarose (FMC Bioproducts) containing PGEM markers (ProMega) as external standards, stained with ethidium bromide, and photographed. The numbers of nucleotide substitutions per site (genetic distances) were estimated by using the method of Nei and Tajima (16). The values were arrayed in a matrix, and a cluster analysis was performed by using the SAHN feature of the NTSYS-pc program (19). A phenogram was then generated by using unweighted pair-group arithmetic average clustering (15). In order to test the goodness of fit of the clustering to the data set, a cophenetic value matrix was constructed (20), and the MXCOMP program was used to compare this matrix with the original matrix.

The PCR products from all of the strains were approximately 1,800 bp long. Selected restriction digests are shown in Fig. 1 through 4. RsaI (Fig. 1) and Taq $\alpha$ I (data not shown) could distinguish only between $S$. inusitatus ATCC $76517^{\mathrm{T}}$ ( $\mathrm{T}=$ type strain) and the other strains. HinfI, HhaI, and ScrfI (Fig. 2 through 4) yielded restriction patterns that separated $S$. inusitatus and $S$. kluyveri from the other strains and divided the remaining strains into two major groups. The other enzymes either did not cut the PCR products or yielded invariable patterns.

The genetic distances obtained by making pairwise comparisons of shared and unique fragments ranged from 0 to 0.059. S. inusitatus ATCC $76517^{\mathrm{T}}$ exhibited the greatest small-subunit rDNA sequence divergence from all of the other strains; this strain was separated from its nearest neighbor ( $S$. kluyveri) by a genetic distance of 0.026 . Both $S$. kluyveri ATCC 76530 and $S$. inusitatus ATCC $76517^{\mathrm{T}}$ occurred alone on separate branches of the phenogram (Fig. 5). The calculated cophenetic correlation coefficient $(0.99)$ indicated that the fit for the cluster analysis was very good (19).

All of the other strains clustered into two groups (Fig. 5). Group 1 ( $S$. bayanus cluster) contained $S$. bayanus, $S$. uvarum, $S$. globosus, $S$. intermedius var. valdensis, $S$. abuliensis, $S$. intermedius var. turicensis, and $S$. mangini var. tetrasporus. Group 2 (S. cerevisiae cluster) contained $S$. tokyo, $S$. cerevisiae subsp. orsati, $S$. cerevisiae subsp. vetrozensis, $S$. ellipsoideus subsp. alpestris, $S$. ellipsoideus subsp. alpinus, $S$. ellipsoideus subsp. fulliensis, $S$. ellipsoideus subsp. thermophilus, $S$. italicus var. melibiosi, and $S$. carlsbergensis. These two groups were separated by a distance of 0.017 , which represented a level of sequence divergence in the small-subunit rDNAs of $1.7 \%$. Within each cluster, the strains produced identical restriction patterns with all of the enzymes. The four subspecies of $S$. ellipsoi- 

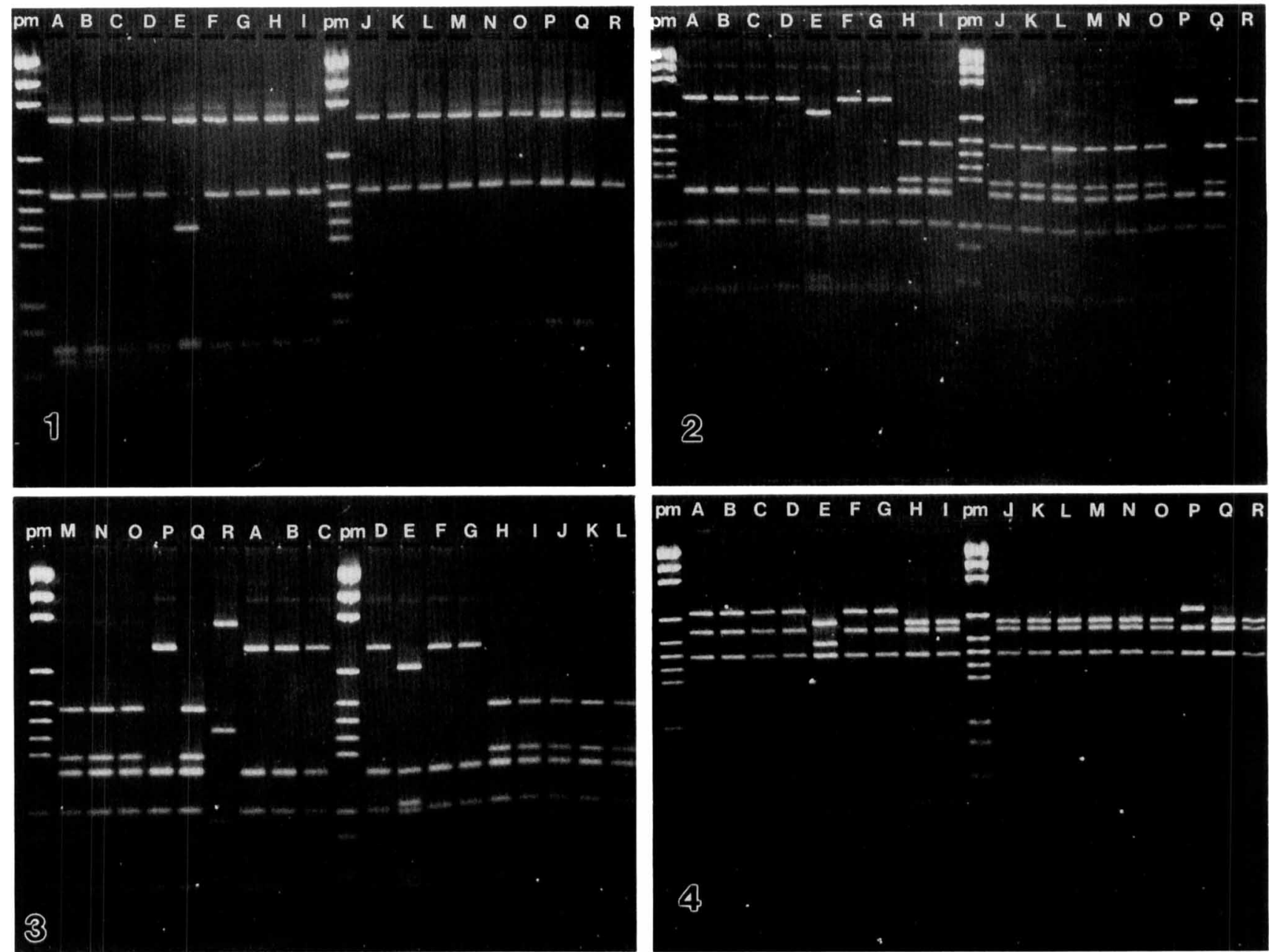

FIG. 1 to 4. Comparison of small-subunit rDNA restriction digests. PCR products were digested with 11 endonucleases, and the resulting fragments were electrophoresed on 2.6\% NuSieve 3:1 agarose. Fig. 1, RsaI digests; Fig. 2, Hinfl digests; Fig. 3, HhaI digests; Fig. 4, ScrfI digests. The PGEM molecular weight standards which we used were $2.64,1.61,1.20,0.68,0.52,0.46,0.40,0.35,0.22,0.18,0.13 \mathrm{~kb}$ long. Lanes pm, PGEM markers; lanes A, S. bayanus ATCC $76513^{\mathrm{T}}$; lanes B, S. uvarum var. uvarum ATCC $76514^{\mathrm{T}}$; lanes C, S. globosus ATCC $76515^{\mathrm{T}}$; lanes D, S. intermedius var. valdensis ATCC $76516^{\mathrm{T}}$; lanes E, S. inusitatus ATCC $76517^{\mathrm{T}}$; lanes F, S. abuliensis ATCC $76518^{\mathrm{T}}$; lanes G, S. intermedius var. turicensis ATCC $76519^{\mathrm{T}}$; lanes H, S. tokyo ATCC $76520^{\mathrm{T}}$; lanes I, S. cerevisiae subsp. orsati ATCC $76521^{\mathrm{T}}$; lanes J, S. cerevisiae subsp. vetrozensis ATCC $76522^{\mathrm{T}}$; lanes K, S. ellipsoideus subsp. alpestris ATCC $76523^{\mathrm{T}} ;$ lanes L, S. ellipsoideus subsp. alpinus ATCC $76524^{\mathrm{T}}$; lanes M, S. ellipsoideus subsp. fulliensis ATCC $76525^{\mathrm{T}}$; lanes N, S. ellipsoideus subsp. thermophilus ATCC $76526^{\mathrm{T}}$; lanes 0 , S. italicus var. melibiosi ATCC $76527^{\mathrm{T}}$; lanes P, S. mangini var. tetrasporus ATCC $76528^{\mathrm{T}}$; lanes Q, S. carlsbergensis ATCC $76529^{\mathrm{T}}$; lanes R, S. kluyveri ATCC 76530.

deus which we examined could not be distinguished from each other on the basis of the 11 digests studied.

Our analysis of restriction polymorphisms in enzymatically amplified small-subunit rDNAs provided further evidence that $S$. cerevisiae Meyen ex Hansen (29) is a genetically heterogeneous group that consists of at least two distinct clusters, one of which corresponds to $S$. bayanus. Similar results were obtained with cloned probes to the $R D N 1$ and HIS4 genes of S. cerevisiae (17). Consistently low nuclear DNA relatedness values have been reported between strains belonging to the $S$. bayanus cluster and $S$. cerevisiae (21, 26, 27). Kaneko and Banno (9) obtained low DNA similarity values for the type strain of $S$. bayanus (strain ATCC 76513) and S. cerevisiae IFO 10217 $7^{\mathrm{T}}$. The fact that these two species are genetically distinct is further indicated by the uniparental segregation of genetic markers in forced-mating studies, suggesting low levels of chromo- some homology (2). These findings support the conclusion that $S$. bayanus should be reinstated as a distinct species (27).

None of the restriction patterns which we obtained permitted differentiation between $S$. carlsbergensis ATCC $76529^{\mathrm{T}}$ and the other species. The occurrence of this strain in the $S$. cerevisiae cluster (this study) is inconsistent with reports of high levels of nucleic acid similarity with $S$. bayanus $(9,10,27)$. However, high levels of DNA relatedness to both $S$. cerevisiae and $S$. bayanus have also been reported (26). $S$. carlsbergensis has been considered a synonym of both species $(27,29)$, and it has been suggested that this organism is a partial amphidiploid that resulted from a rare mating (26).

The occurrence of $S$. kluyveri on a branch of the phenogram that is separated from the $S$. cerevisiae cluster is consistent with previously reported differences in the chro- 
TABLE 1. Strains of $S$. cerevisiae sensu Yarrow examined

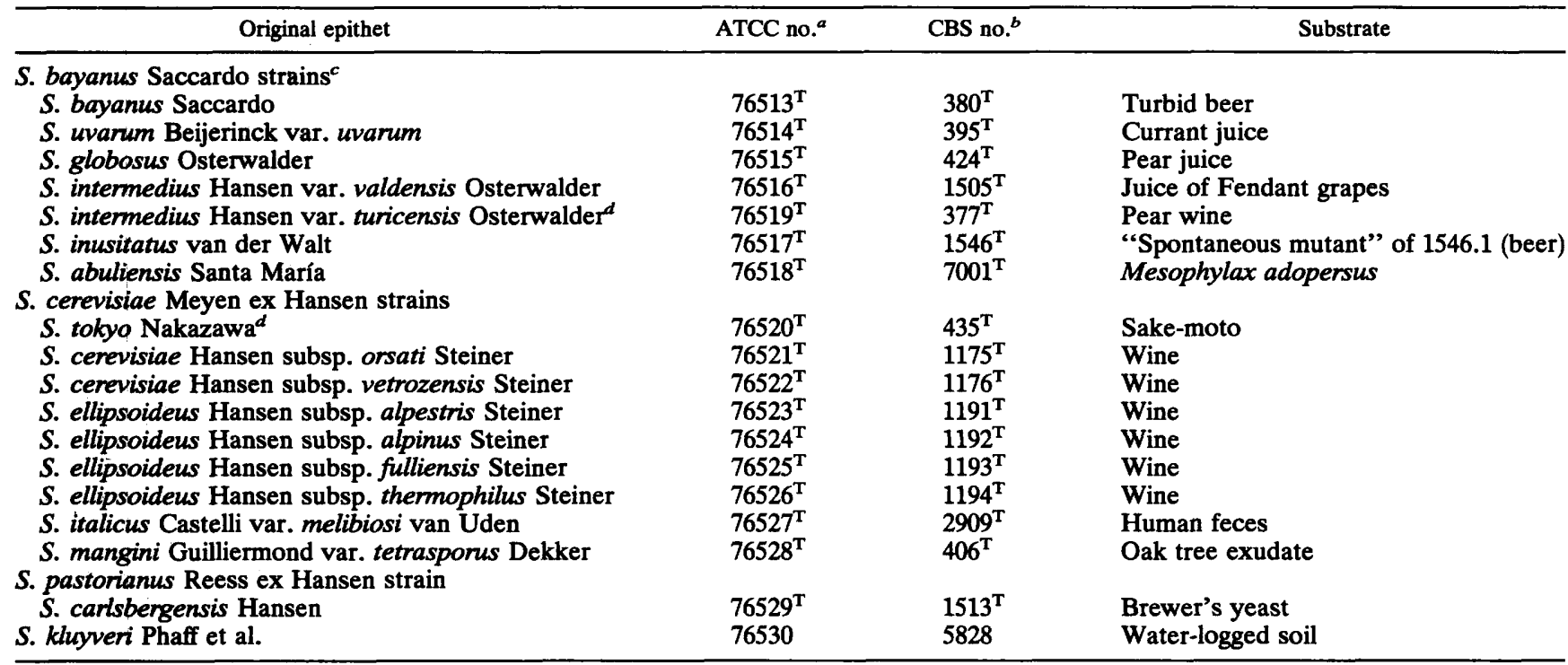

a ATCC, American Type Culture Collection.

b CBS, Centraalbureau voor Schimmelcultures.

c Based on the nomenclature of Vaughan-Martini and Martini (27) and Naumov (14).

d Affinities have not been determined previously by molecular methods.

mosome numbers of these organisms ( 5 in $S$. kluyveri versus 14 to 17 in $S$. cerevisiae) (8). This distinction also correlates well with the large rRNA sequence divergence between these species (10) and with differences in their cellular fatty acid profiles (1). Thus, there appears to be overwhelming support for the concept that $S$. kluyveri is an outlying member of the genus Saccharomyces (10).

We did not expect to find remarkably unique restriction patterns for $S$. inusitatus ATCC $76517^{\mathrm{T}}\left(=\mathrm{CBS} 1546^{\mathrm{T}}\right)$ since a high level $(94 \%)$ of nuclear DNA reassociation between this strain and the type strain of $S$. bayanus has been reported previously (26). Although strain ATCC $76517^{\mathrm{T}}$ has been described as a spontaneous mutant (6), it is possible that this organism was actually an unrelated contaminant. Therefore, we examined its parent strain, $S$. inusitatus CBS

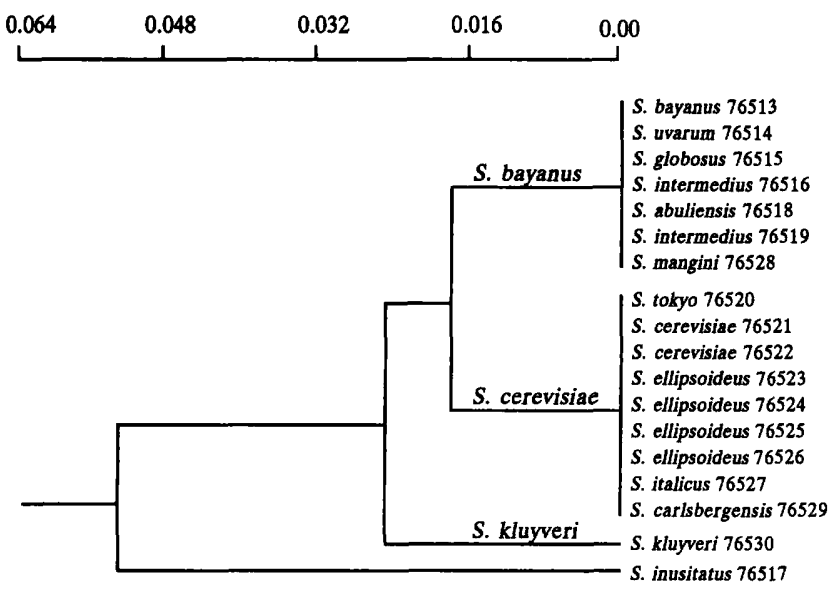

FIG. 5. Phenogram generated from unweighted average pair group clustering of genetic distances between strains. The strain designations are American Type Culture Collection (ATCC) numbers.
1546.1, and found that the resulting profiles were indeed identical to the restriction patterns of the strains belonging to the $S$. bayanus cluster and did not resemble the profile of the derived culture (strain ATCC $76517^{\mathrm{T}}$ ).

Correlation of our results with previous findings $(9,21,26$, 27 ) showed that $S$. bayanus is quite distinct from $S$. cerevisiae. We are presently investigating the extent of interstrain variation in restriction fragment length polymorphisms in this species complex. We are also examining the region that spans the two internal transcribed spacers and the $5.8 \mathrm{~S}$ rDNA to determine whether we can obtain sufficient resolution to differentiate the two other distinct taxa in this species complex, $S$. pastorianus and $S$. paradoxus.

This study was supported in part by grant DIR89-15137 from the National Science Foundation.

We thank W. Siegel for technical assistance in the preparation of the figures.

\section{REFERENCES}

1. Augustyn, O. P. H., D. Ferreira, and J. L. F. Kock. 1991. Differentiation between yeast species, and strains within a species, by cellular fatty acid analysis. 4 . Saccharomyces sensu stricto, Hanseniaspora, Saccharomycodes and Wickerhamiella. Syst. Appl. Microbiol. 14:324-334.

2. Banno, I., and Y. Kaneko. 1989. A genetic analysis of taxonomic relation between Saccharomyces cerevisiae and Saccharomyces bayanus. Yeast 5(Special Issue):S373-S377.

3. Barnett, J. A., R. W. Payne, and D. Yarrow. 1990. Yeasts: characteristics and identification, p. 594-602. Cambridge University Press, Cambridge.

4. Bicknell, J. N., and H. C. Douglas. 1970. Nucleic acid homologies among species of Saccharomyces. J. Bacteriol. 101:505512.

5. Campbell, I. 1972. Numerical analysis of the genera Saccharomyces and Kluyveromyces. J. Gen. Microbiol. 73:279-301.

6. Centraalbureau voor Schimmelcultures. 1990 . List of culturesfungi and yeasts, p. 359-365. Institute of the Royal Netherlands Academy of Arts and Sciences, Baarn, The Netherlands.

7. Gilliland, R. B. 1969. The raffinose fermentation of Saccharo- 
myces pastorianus and Saccharomyces bayanus. Antonie van Leeuwenhoek 35:13-23.

8. Johnston, J. R., and R. K. Mortimer. 1986. Electrophoretic karyotyping of laboratory and commercial strains of Saccharomyces and other yeasts. Int. J. Syst. Bacteriol. 36:569-572.

9. Kaneko, Y., and I. Banno. 1991. Reexamination of Saccharomyces strains by DNA-DNA hybridization and electrophoretic karyotyping. IFO Res. Commun. 15:30-41.

10. Kurtzman, C. P., and C. J. Robnett. 1991. Phylogenetic relationships among species of Saccharomyces, Schizosaccharomyces, Debaryomyces and Schwanniomyces determined from partial ribosomal RNA sequences. Yeast 7:61-72.

11. Medlin, L., J. Elwood, S. Stickel, and M. L. Sogin. 1988. The characterization of enzymatically amplified eukaryotic 16S-like ribosomal RNA sequences for phylogenetic analyses. Proc. Natl. Acad. Sci. USA 82:6955-6959.

12. Molina, F. I., T. Inoue, and S.-C. Jong. 1992. Determination of infraspecific relationships in Kluyveromyces marxianus by riboprinting. Mycotaxon 43:49-60.

13. Naumov, G. I. 1972. Revision of the classification of the Saccharomyces yeasts on the basis of genetic analyses, p. 34-38. In Second Congress of the All-Union Society of Geneticists and Selectionists, vol. 1. General Molecular Genetics. USSR Academy of Sciences, Moscow.

14. Naumov, G. I. 1987. Genetic basis for classification and identification of the ascomycetous yeasts. Stud. Mycol. 30:469-475.

15. Nei, M. 1987. Molecular evolutionary genetics. Columbia University Press, New York.

16. Nei, M., and F. Tajima. 1983. Maximum likelihood estimation of the number of nucleotide substitutions from restriction site data. Genetics 105:207-217.

17. Pedersen, M. B. 1983. DNA sequence polymorphisms in the genus Saccharomyces. I. Comparison of the His 4 and ribosomal RNA genes in lager strains, ale strains and various species. Carlsberg Res. Commun. 48:485-503.

18. Price, C. W., G. B. Fuson, and H. J. Phaff. 1978. Genome comparison in yeast systematics: delimitation of species within the genera Schwanniomyces, Saccharomyces, Debaryomyces, and Pichia. Microbiol. Rev. 42:161-193.
19. Rohlf, F. J. 1990. NTSYS-pc: numerical taxonomy and multivariate analysis system, p. 7.6-7.10. Exeter Publishing, Ltd., New York.

20. Rohlf, F. J., and R. R. Sokal. 1981. Comparing numerical taxonomic studies. Syst. Zool. 17:407-412.

21. Rossini, G. F., F. Federici, A. E. Vaughan, and A. Martini. 1982 Systematics of the yeast genus Saccharomyces associated with the fermentation industry. Eur. J. Appl. Microbiol. Biotechnol. 15:188-193.

22. Scheda, R., and D. Yarrow. 1966. The instability of physiological properties used as criteria in the taxonomy of yeasts. Arch. Mikrobiol. 55:209-225.

23. Scheda, R., and D. Yarrow. 1968. Variation in the fermentative pattern of some Saccharomyces species. Arch. Mikrobiol. 61: 310-316.

24. van der Walt, J. P. 1970. Saccharomyces Meyen emend. Reess, p. 555-718. In J. Lodder (ed.), The yeasts: a taxonomic study. Elsevier/North-Holland Publishing Co., Amsterdam.

25. Vaughan-Martini, A. 1989. Saccharomyces paradoxus comb. nov., a newly separated species of the Saccharomyces sensu stricto complex based upon nDNA/nDNA homologies. Syst. Appl. Microbiol. 12:179-182.

26. Vaughan-Martini, A., and C. P. Kurtzman. 1985. Deoxyribonucleic acid relatedness among species of the genus Saccharomyces sensu stricto. Int. J. Syst. Bacteriol. 35:508-511.

27. Vaughan-Martini, A., and A. Martini. 1987. Three newly delimited species of Saccharomyces sensu stricto. Antonie van Leeuwenhoek J. Microbiol. 53:77-84.

28. White, T. J., T. Bruns, S. Lee, and J. Taylor. 1990. Amplification and direct sequencing of fungal ribosomal RNA genes for phylogenetics, p. 315-322. In M. A. Innis, D. H. Gelfand, J. J. Sninsky, and T. J. White (ed.), PCR protocols. Academic Press, Inc., San Diego, Calif.

29. Yarrow, D. 1984. Saccharomyces Meyen ex Reess, p. 379-395. In N. J. W. Kreger-van Rij (ed.), The yeasts: a taxonomic study. Elsevier Science Publishing, Inc., New York.

30. Yarrow, D., and T. Nakase. 1975. DNA base composition of species of the genus Saccharomyces. Antonie van Leeuwenhoek J. Microbiol. Serol. 41:81-88. 